LONG COURSE ARTICLE

\title{
Acute lung injury-from cannabis to COVID
}

\author{
Mary Beth Beasley (iD ${ }^{1 凶}$ \\ (C) The Author(s), under exclusive licence to United States \& Canadian Academy of Pathology 2021
}

Recent world events have refocused attention on the pathology associated with clinical acute respiratory distress syndrome (ARDS). The vast majority of cases of clinical ARDS will have diffuse alveolar damage (DAD) histologically, but other histologies may occur less frequently. The aim of this paper is to provide a review of the pathology of DAD and acute fibrinous and organizing pneumonia and provide insights into the pathologic features associated with the E-cigarette/vaping-associated lung-injury outbreak and the ongoing SARS-CoV-2 pandemic.

Modern Pathology (2022) 35:1-7; https://doi.org/10.1038/s41379-021-00915-6

\section{INTRODUCTION}

The outbreak of E-cigarette/vaping-associated lung injury (EVALI) in the fall of 2019, followed closely by the onset of the catastrophic global pandemic due to severe acute respiratory syndrome coronavirus-2 (SARS-CoV-2) infection, still ongoing at the time of this writing, has resulted in increased focus on the pathology of acute-onset lung disease associated with the clinical scenario of acute respiratory distress syndrome (ARDS).

Broadly, acute pulmonary injury may result from myriad direct or indirect pulmonary insults, often culminating in hypoxemic respiratory failure ${ }^{1}$. From a clinical standpoint, "acute lung injury" (ALI) was formally defined in the 1994 American-European Consensus Conference as part of the definition of ARDS. In this classification, ARDS was defined as the presence of acute hypoxemia with (1) a PaO2:FiO2 ratio of $200 \mathrm{mmHg}$ or less, (2) bilateral infiltrates radiographically consistent with pulmonary edema, and (3) no clinical evidence of cardiac failure. ALI was defined by the same criteria only with a PaO2:FiO2 of $300 \mathrm{mmHg}^{2}$. Subsequently, the Berlin definition of ARDS included (1) timing of disease within one week of known insult or worsening respiratory symptoms, (2) chest imaging with bilateral opacities not fully explained by effusions, lobar/lung collapse, or nodules, and (3) origin of edema not explained by cardiac failure or fluid overload, as well as a need for objective assessment such as echocardiography to exclude hydrostatic edema if no risk factor was present. The Berlin definition eliminated the category of ALI and instead divided ARDS into three categories: mild $\left(\mathrm{PaO}_{2}: \mathrm{FiO}_{2}<200 \mathrm{mg}\right.$, $\leq 300 \mathrm{mg}$ with PEEP, or CPAP $\left.\geq 5-\mathrm{cm} \mathrm{H}_{2} \mathrm{O}\right)$, moderate $\left(\mathrm{PaO}_{2}: \mathrm{FiO}_{2}<\right.$ $100 \mathrm{mg}, \leq 200 \mathrm{mg}$ with PEEP $\left.\geq 5-\mathrm{cm} \mathrm{H}_{2} \mathrm{O}\right)$, and severe $\left(\mathrm{PaO}_{2}: \mathrm{FiO}_{2}<\right.$ $100 \mathrm{mg}$, with PEEP $\left.\geq 5-\mathrm{cm} \mathrm{H}_{2} \mathrm{O}\right)^{3}$. The updated Kigali modification retains essentially the same definitions as the Berlin definition; however, the $\mathrm{PaO}_{2}: \mathrm{FiO}_{2}$ ratio was modified to $\leq 315 \mathrm{mmHg}$ and the PEEP requirement was eliminated. In the updated schemes, clinical ALI roughly corresponds to the new category of "mild" ARDS. The goal of these definitions was to provide a uniform basis for diagnosis and reporting; however, ARDS has long been recognized as being more clinically heterogeneous than these definitions would suggest, and various subtypes and endotypes have long been recognized ${ }^{1,4-6}$. The mortality rate for clinical ARDS has historically been reported as being up to $60 \%$, with a higher mortality rate in older individuals; however, other studies have reported a mortality rate of $40 \%$ for severe ARDS and a lower percentage for mild or moderate disease ${ }^{7}$.

From a pathologic standpoint, the histology associated with a clinical presentation of ALI/ARDS is typically diffuse alveolar damage (DAD). Other disorders encountered in this clinical setting may include acute fibrinous and organizing pneumonia (AFOP), acute eosinophilic pneumonia (AEP), and, occasionally, diffuse alveolar hemorrhage with capillaritis (DAHC). Organizing pneumonia (OP) is included in the ATS/ERS classification of idiopathic interstitial pneumonias under the heading of "acute/subacute interstitial pneumonias" and, while useful to keep in mind when approaching a biopsy from a patient with a relatively short duration of disease, most cases of OP do not present with clinical ALI/ARDS. It should also be noted that acute exacerbation may occasionally be the initial presentation of a chronic interstitial lung disease such as usual interstitial pneumonia ${ }^{8,9}$.

In keeping with the USCAP long course lecture, the focus of this article will be DAD and AFOP and the pulmonary findings in EVALI and COVID-19. The interested reader is referred to several comprehensive articles on AEP and diffuse alveolar hemorrhage $^{10-12}$.

\section{DAD}

$D A D$ is the most frequent histologic finding in patients with clinical ARDS. The SARS-CoV-2 pandemic has provided an opportunity to apply new methodologies to the study of the pathogenesis of DAD, which will be discussed in the section on COVID. The disease process can briefly be summarized as injury to the capillary endothelium and alveolar epithelium, which have a fused basement membrane in parts of the alveolar wall, resulting in the exudation of fluid and cellular-breakdown products into the alveolar spaces, which is followed by a reparative phase characterized by type- 2 pneumocyte hyperplasia and fibroblastic proliferation $^{8,9,13,14}$.

\footnotetext{
${ }^{1}$ Department of Pathology, Molecular and Cell-based Medicine, Icahn School of Medicine at Mount Sinai, New York, NY, USA. ${ }^{凶}$ email: Mary.beasley@mountsinai.org
} 


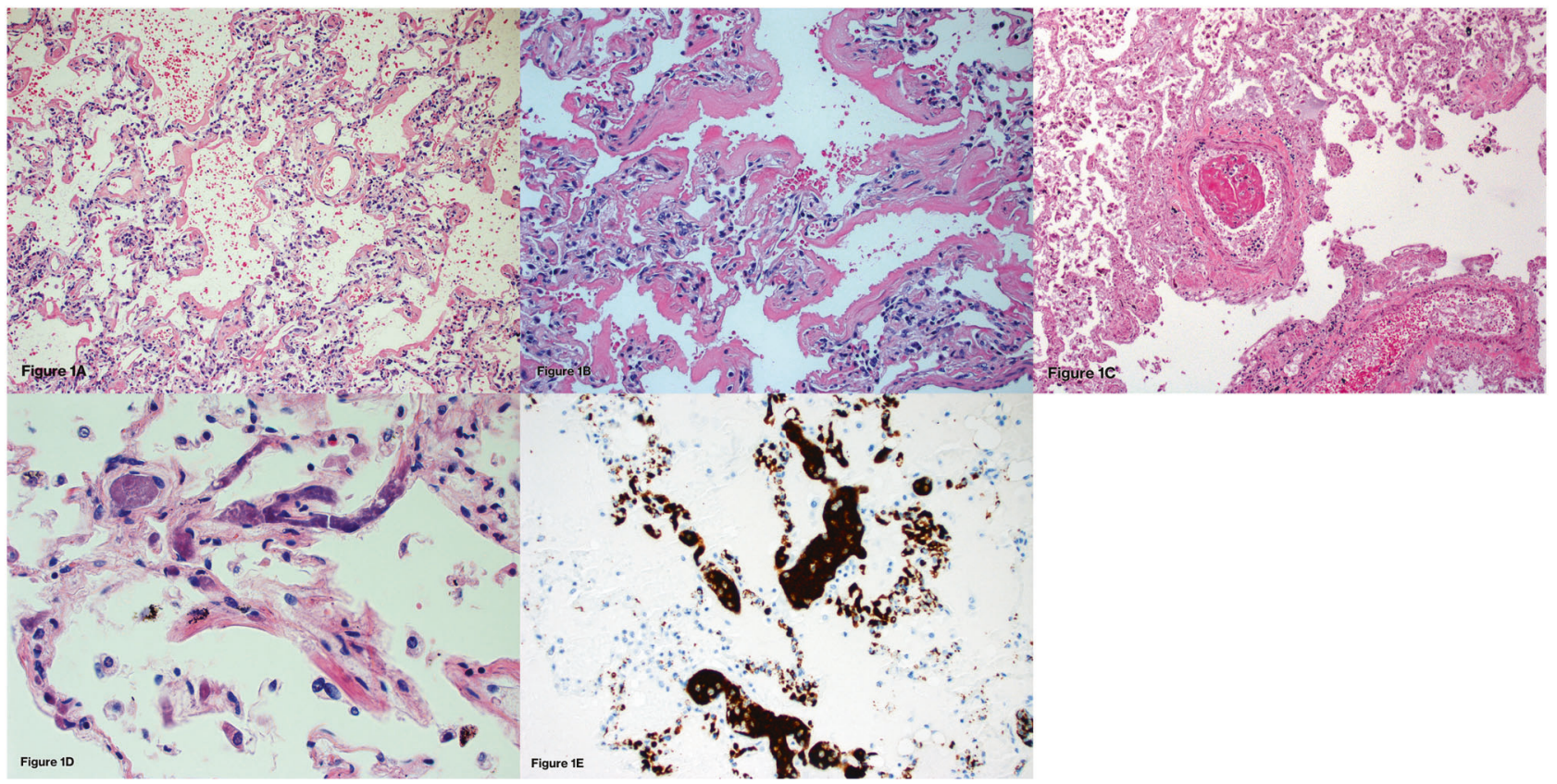

Fig. 1 Acute diffuse alveolar damage (DAD). A Low power of acute DAD showing slight residual edema and condensation of hyaline membranes along alveolar walls (H\&E 100x). B Hyaline membranes characteristic of the acute phase of DAD have a glassy, hyalinized appearance and are composed of proteinaceous material and cellular debris (H\&E 200x). C Fibrin thrombi may be present in cases of acute DAD, regardless of etiology, and are more frequently seen in the acute phase (H\&E 100x). D Platelet aggregates and thrombi may also be observed in cases of DAD and consist of pink-to-purple granular material within blood vessels (H\&E 200X). E Platelet aggregates highlighted by CD61 staining (CD61 200x).

DAD is classically divided into acute/exudative, organizing/ proliferative, and fibrotic phases. The acute/exudative phase roughly corresponds to the first seven days following initial insult, the organizing/proliferative phase to days $7-21$, and the fibrotic phase occurring after 21 days. Following initial insult, the earliest changes can be seen only at the ultrastructural level. By day 2, intra-alveolar edema and interstitial widening become apparent. Hyaline membranes, composed of cellular and proteinaceous debris, begin to appear at this point and reach a peak around days 4-5. Hyaline membranes have a glassy eosinophilic appearance and characteristically line alveolar ducts and alveolar walls (Fig. 1A, $B)$. Occasional cases may have prominent intra-alveolar-organizing fibrin in addition to hyaline membranes. In spite of the proposed role of inflammation in the pathogenesis of DAD, inflammatory-cell infiltrates are typically sparse. Fibrin thrombi may be present and are often quite extensive (Fig. 1C). Platelet thrombi may also be present (Fig. 1D, E). These thrombi are secondary to localized activation of the coagulation pathway as part of the evolving repair process and should not be taken as evidence of an underlying thromboembolic disorder. The vascular component of DAD has received a great deal of attention in the COVID literature and will be discussed further in that section. DAD classically involves the lung in a relatively diffuse fashion; however, the name "diffuse" refers to diffuse injury of the alveolar wall and, as such, the disease may not always involve the lung itself in a diffuse fashion ${ }^{8,9,13-15}$.

While the acute/exudative phase of DAD is generally readily recognizable due to the presence of characteristic hyaline membranes, the organizing/proliferative phase may prove more problematic from a diagnostic standpoint histologically. This phase is characterized by interstitial fibrosis associated with pronounced type 2 pneumocyte hyperplasia. Hyaline membranes are resorbed into the interstitium and may be present only focally or absent entirely. The pneumocyte hyperplasia may show quite marked cytologic atypia and mitotic figures may occasionally be present. The pneumocytes respect the alveolar architecture but typically maintain a hobnail pattern of growth and do not show the continuous linear growth, cellular stratification or the abrupt demarcation from uninvolved lung typically encountered in lepidic growth of carcinoma. Squamous metaplasia may also be present, which should similarly not be confused with malignancy but, again, the overall disease context and distribution should aid in this distinction. The fibrosis present in DAD typically has a loose myxoid appearance that appears bluish-gray on $\mathrm{H} \& \mathrm{E}$ sections (Fig. 2A-D). This is in contrast to the collagenous fibrosis encountered in chronic interstitial lung diseases such as usual interstitial pneumonia and nonspecific interstitial pneumonia. Plugs of organizing fibrous tissue may occlude the alveolar ducts (alveolar-duct fibrosis). Macrothrombi and obliterative endarteritis may also be seen in this phase $e^{8,9,13-15}$.

If a patient survives for more than three weeks following initial insult, the myxoid fibrosis becomes replaced by collagenous fibrosis and may show irregular enlargement of airspaces or microscopic honeycombing. Traction bronchiectasis may also be present. Vascular changes such as mural fibrosis and medial hypertrophy may be present ${ }^{13-15}$.

As stated, DAD may be the result of myriad pulmonary insults and, unfortunately, absent identification of an infectious agent, the etiology cannot otherwise be determined by the histology alone. Cases in which a patient has DAD histologically and idiopathic disease clinically are assigned the clinicopathologic designation "Acute Interstitial Pneumonia (AIP)" using the current guidelines for idiopathic interstitial pneumonias. AIP is not a pathologic diagnosis ${ }^{16}$.

The histologic differential diagnosis of DAD includes primarily other disorders associated with clinical ARDS, namely AFOP, AEP, and DAHC. As discussed below, while some cases of DAD may have prominent organizing intra-alveolar fibrin, the presence of hyaline membranes is diagnostic of DAD and excludes a diagnosis of AFOP. The presence of eosinophils in a case otherwise resembling DAD supports a diagnosis of AEP and eosinophils should be sought in all cases, given that AEP is typically responsive to steroid therapy. Finally, some cases of DAD may have prominent 


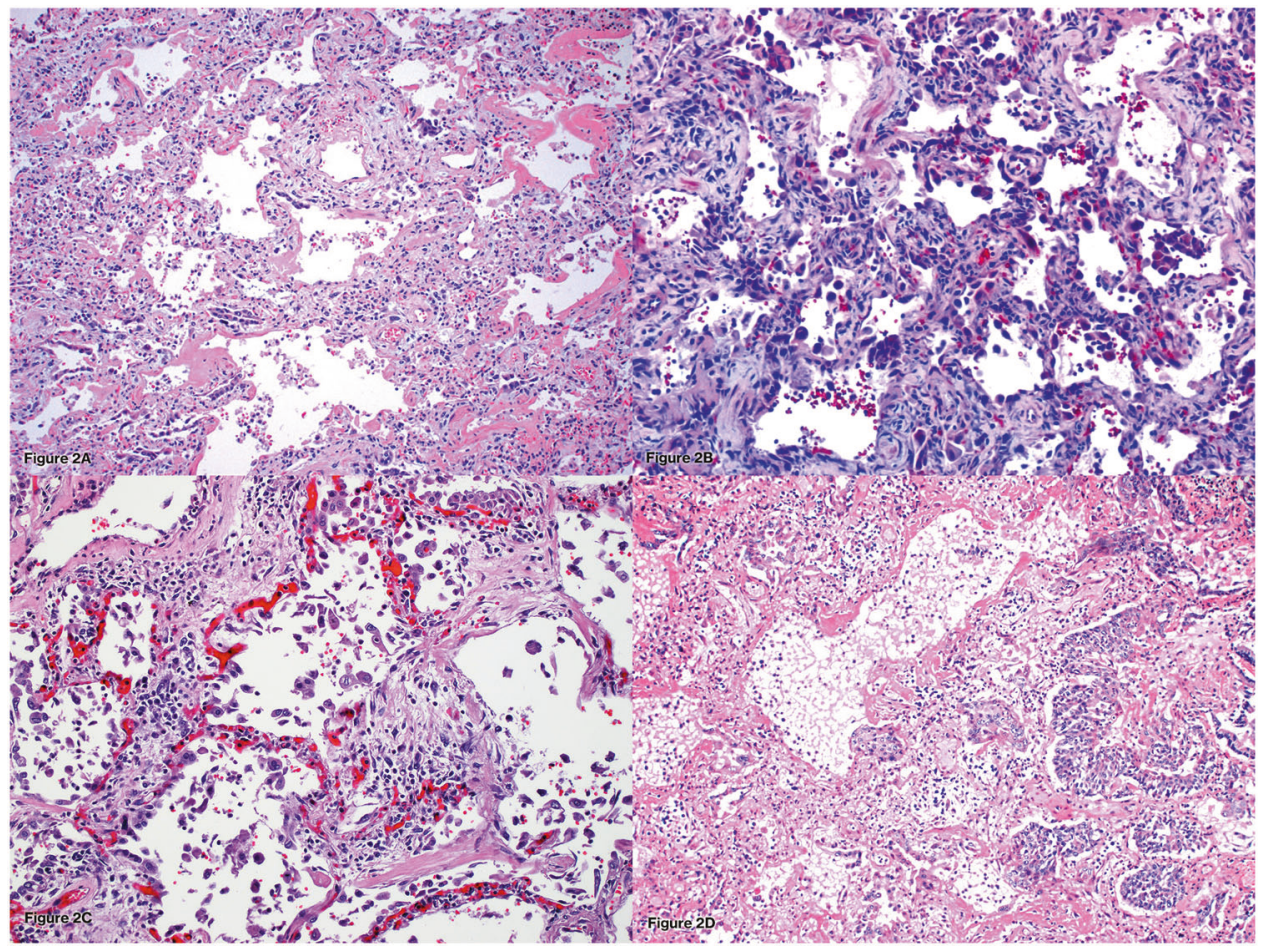

Fig. 2 Organizing diffuse alveolar damage. A Early organizing DAD showing residual hyaline membranes. The interstitium is expanded by bluish-gray myxoid fibrosis (H\&E 200x). B Organizing DAD showing myxoid interstitial fibrosis and pronounced type-2 pneumocyte hyperplasia (H\&E 200x). C Pneumocyte hyperplasia may show multinucleation and marked cytologic atypia (H\&E200x). D Squamous metaplasia may also be present in cases of organizing diffuse alveolar damage, in this case, residual hyaline membranes are also present (H\&E 100x).

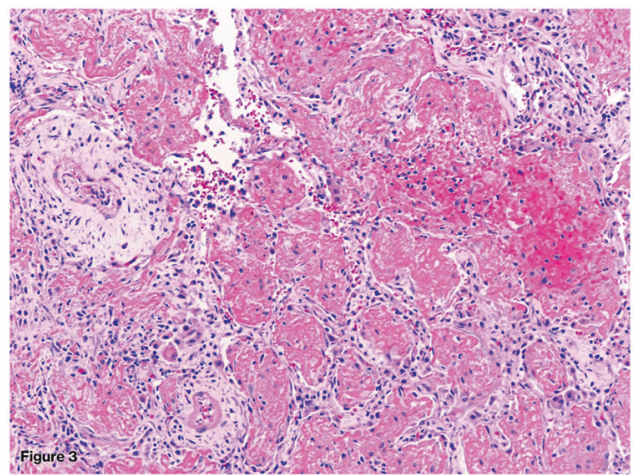

Fig. 3 Acute Fibrinous and Organizing Pneumonia. In contrast to $\mathrm{DAD}$, cases of AFOP demonstrate intra-alveolar-organizing fibrin balls and lack hyaline membrane formation (H\&E 100x).

hemorrhage and features of vasculitis or capillaritis should be sought in such cases to exclude a primary vasculitic process ${ }^{8,9}$.

\section{AFOP}

AFOP was originally described in 2002 by Beasley et al. as a histologic pattern associated with an acute or subacute disease onset that did not meet the histologic criteria of $D A D, A E P$, or $O P$, but rather consisted of organizing intra-alveolar fibrin balls without hyaline membrane formation, significant neutrophils, eosinophils, or macrophages (Fig. 3). In the original study of 17 cases, the disease typically had a patchy distribution, although some cases were relatively diffuse with a range of $25-90 \%$ airspace involvement. The amount of fibrin was not explicitly specified aside from the fact that it was the "dominant" finding, and varying degrees of organizing pneumonia were also present. The overall mortality rate was similar to DAD; however, it should be noted that in this study, there was in reality a bimodal survival distribution with nine patients dying of disease in 36 days or less from disease onset, while seven patients had less severe, indolent disease and recovered and one patient died of unrelated causes. Similar to other interstitial lung diseases, over half of the cases in this study were felt to be associated with an underlying etiology such as collagen vascular disease, occupational exposure, drug reaction, or infection ${ }^{17}$. Since this original report, AFOP has remained a relatively rare diagnosis, with reported cases largely limited to individual case reports or small series. Gomes et al. reported a series of 13 cases, 11 of which had underlying etiologies and a median survival of 78 months with seven patients dying and six alive at the time of publication ${ }^{18}$. In viewing the overall reported literature, there continues to be a variable survival with some cases acting in a similar fashion to DAD with a high mortality, while others have an indolent course. AFOP has been reported with a wide range of underlying etiologies such as viral infections (SARS-CoV-2 will be discussed below), drug reactions including checkpoint inhibitors, collagen vascular diseases, and following hematopoietic stem cell transplantation. AFOP has also been reported as a histologic pattern of acute hypersensitivity pneumonitis and as a proposed form of lung allograft rejection ${ }^{19-27}$. As with $D A D$, unless an infectious etiology is identified, the underlying cause cannot be determined by the histology alone. The ATS/ERS classification of idiopathic interstitial lung disease regards AFOP as a "rare histologic pattern" but it is not classified as a "stand alone" disease entity ${ }^{16}$. Indeed, some cases of AFOP would seem to represent a variant of DAD, while other cases would appear to represent a possible variant of OP.

As stated, the histology of AFOP consists of a predominant fibrin or intra-alveolar fibrin balls. Hyaline membranes, significant neutrophils, and eosinophils are lacking. The interstitium may show sparse chronic inflammation or myxoid expansion similar to that described in DAD. An important caveat regarding AFOP is that, similar to many interstitial lung diseases, a definitive diagnosis 
requires a large specimen such as a wedge biopsy and should not be made on a small biopsy such as a transbronchial biopsy or core specimen. The reason for this is twofold. First, as previously mentioned, some cases of DAD may have prominent intra-alveolar fibrin and a small specimen may miss hyaline membranes otherwise diagnostic of DAD. Second, a similar issue with sampling may occur with a small biopsy as intra-alveolar fibrin may be found as a secondary reaction either adjacent to or as part of another disease process. Alveolar fibrin may particularly be found adjacent to necrotizing granulomas or abscesses in particular, but may also be adjacent to neoplastic processes. Organizing fibrin may also be found in association with acute bacterial pneumonias or vasculitic processes. As such, careful clinical and radiologic correlation is required to determine the significance of organizing intra-alveolar fibrin in a small biopsy $8,9,17$.

The differential diagnosis of AFOP primarily includes DAD, eosinophilic pneumonia (EP), and OP. As previously stated, some cases of DAD may have prominent alveolar fibrin; however, if hyaline membranes are present, the case should be diagnosed as DAD and not AFOP. Interestingly, a study of SARS-CoV-1 (SARS-1) cases from Toronto in 2005 describes cases of AFOP ${ }^{28}$. The cases included in the "AFOP pattern" or "mixed AFOP and DAD patterns" in this study would properly fall under the category of DAD based on current knowledge; however, the findings do raise the issue of whether this disease pattern has any particular clinical significance.

Eosinophilic pneumonia frequently has prominent intra-alveolar fibrin and may greatly resemble AFOP; however, the presence of eosinophils excludes a diagnosis of AFOP. Further, where studied, AFOP has not been associated with peripheral blood eosinophilia that is typically present in EP, although it may be absent in AEP. Eosinophils may disappear from tissue quickly following administration of steroids, so a partially treated EP should be excluded clinically ${ }^{9,12}$.

Discrimination of OP from AFOP depends largely on the presence of fibrin. While the presence of a predominant component of intra-alveolar fibrin in a case containing areas of OP may be more readily diagnosed as AFOP, the converse issue of how much fibrin should be present in a case otherwise consisting primarily of OP before a diagnosis of AFOP is made is less clear, although an important issue given the potential clinical implications. The presence of fibrin has long been associated with an increased incidence of relapse after treatment of OP and, in 1995, Yoshinouchi et al. described what the authors called "Type 2 COP," which featured a fibrin component and was associated with an increased incidence of relapse ${ }^{29}$. Nishino et al. evaluated the pathology from 26 patients with clinical cryptogenic-organizing pneumonia (COP) and divided the cases into those with no fibrin, focal fibrin $(<0.2-\mathrm{cm}$ greatest-dimension focus in one histologic section, multifocal (multiple foci involving more than one histologic section and AFOP (ball-like aggregates diffusely filling alveoli). In this study, cases with multifocal fibrin and AFOP had relapse rates of $43 \%$ and $100 \%$, respectively, while those with focal fibrin had one relapse and those with no fibrin had none. This study additionally demonstrated that patients with disease involving at least three lung zones also had higher rates of relapse $^{30}$. While these studies suggest that more fibrin and more extensive disease are important predictors of relapse, a definitive minimal threshold has not been elucidated. From a practical standpoint, the presence of any fibrin in a case otherwise consistent with OP should be mentioned, given the increased potential for relapse following therapy.

\section{E-cigarette/vaping-associated acute lung injury (EVALI)}

In mid-2019, an outbreak of severe respiratory disease associated with the use of various vaping products received widespread media attention. The increase in cases was unrelated to an increase in vaping in general or increased recognition of disease, suggesting a new component/contaminant or behavior. A study by Layden et al. reported the findings in 53 cases occurring in Illinois and Wisconsin, which contributed to the establishment of the CDC guidelines for cases of severe pulmonary disease associated with e-cigarette use ${ }^{31}$. Ultimately, the majority of cases were found to be primarily associated with the use of THC-based products, particularly those from black-market sources. For this particular outbreak, vitamin E acetate oil, a substance added to THC-containing products as a cutting agent, was identified as the likely inciting component, given the tendency for this oil to transform into a substance similar to phosgene gas when heated to high temperature ${ }^{32,33}$. Once this cutting agent was eliminated, cases of EVALI associated with that outbreak decreased; however, vaping products contain a wide range of components, typically nicotine or THC admixed with various suspension solutions or flavors, many of which are unregulated, so cases continue to be reported. Additionally, other components of the vaping device have the potential to produce lung damage, as evidenced by one case of giant-cell interstitial pneumonia traced to cobalt in a heating coil ${ }^{34}$.

Initially, the pathology of EVALI was poorly defined, with many reports lacking tissue pathology, adequate descriptions, or images. The previously mentioned Layden et al. article reported pathology from 17 of the 53 cases, which included 14 BALs and three transbronchial biopsies. Seven of the BALs reported lipid-laden macrophages and the tissue biopsies showed DAD, interstitial and peribroncial granulomatous pneumonitis, and foamy macrophages $^{31}$. The presence of lipid-laden macrophages in the BALs in this study and other reports gave rise to the concept that EVALI was characterized by lipoid pneumonia, and that lipid-laden macrophages in BAL-fluid were diagnostic of the disease. This caused some confusion, given that the radiology of reported EVALI cases did not resemble lipoid pneumonia and lipid-laden macrophages in BAL fluid are largely a nonspecific finding in BALfluid specimens not related to EVALI ${ }^{35}$. Two histologic studies were published in close succession-Butt et al. and Mukhopadyayy et al. reported 17 cases and eight cases of EVALI, respectively ${ }^{36,37}$. Both studies reported similar findings, consisting of various histologic patterns associated with acute or subacute lung injuries such as DAD, OP, or AFOP. Some of the cases did contain foamy macrophages; however neither study demonstrated findings of exogenous lipid pneumonia. As such, while vacuolated macrophages were seen in a subset of cases, they were not present in all cases and should not be used as definitive support for EVALI. Subsequent studies have also reported similar findings of DAD, AFOP, and OP in addition to AEP and diffuse alveolar hemorrhage ${ }^{38,39}$. Ultimately, as with all cases of histologic DAD or other acute lung-injury patterns, clinical and radiographic correlation is needed to aid in determining etiology.

\section{SARS-CoV-2/COVID-19}

The coronavirus disease-2019 (COVID-19) pandemic caused by the beta-coronavirus severe acute respiratory syndrome coronavirus 2 (SARS-CoV-2) has claimed nearly 4.5 million lives worldwide and is still ongoing at the time of this writing. While other organs may be affected, the majority of patients suffer from respiratory symptoms and expire from respiratory failure. The purpose of this section is not to provide a comprehensive review of all components of lung disease in COVID-19 patients, but to focus on the major histologic findings and areas of particular interest from a pathology standpoint.

Information regarding the pathology associated with SARS-CoV2 infection has thus far been derived predominantly from autopsy studies. DAD has been the reported pulmonary finding in the overwhelming majority of cases ${ }^{40-52}$. Larger series of cases from my own institution of Mt. Sinai, presented in the study of Bryce et al. and incorporated as a component of the cases in Borczuk et al., showed DAD in the majority of cases. These series were both from early in the pandemic and DAD was primarily in the acute phase with a smaller component in the organizing phase, with the 


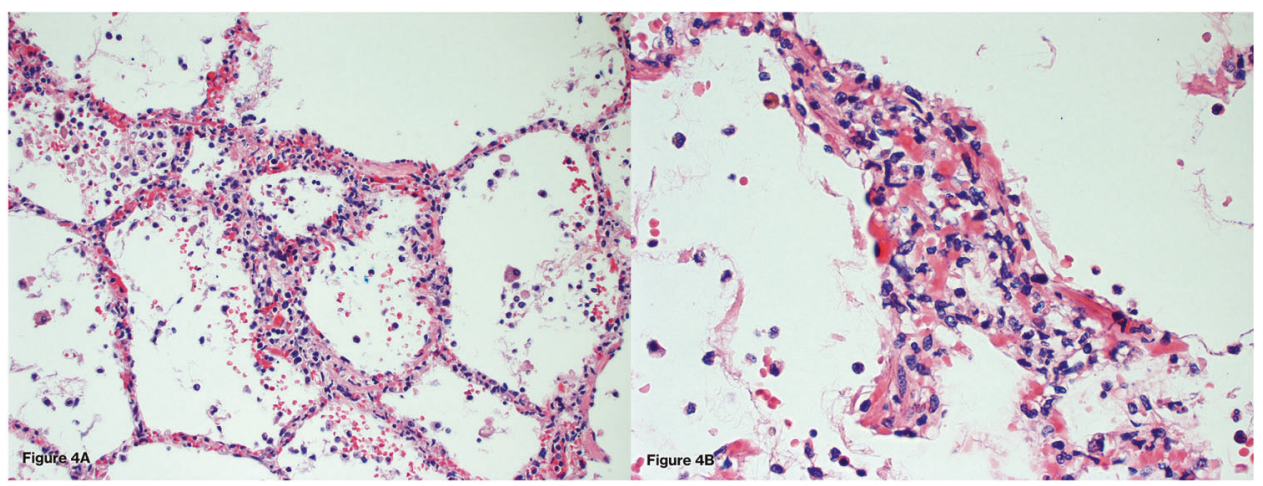

Fig. 4 Vascular abnormalities in cases of COVID-19 DAD. Some cases of COVID-19 DAD contained foci suggesting vascular damage/ endothelitis (A H\&E 40x and B H\&E 200x). Such foci were felt to represent early changes and likely represent an underreported finding in DAD as opposed to a unique feature of COVID-related disease.

latter occurring in patients with longer survival, as would be expected ${ }^{41,42}$. In the study of Borczuk et al., in particular, the large airways showed acute ulcerative lesions in some cases and airway epithelium similarly showed evidence of injury or regeneration ${ }^{41}$. Superimposed acute pneumonia was seen in 45 cases in the series of Bryce et al., which was primarily seen in patients with longer disease duration ${ }^{42}$. In other series, progression to the fibrotic phase of DAD was also reported, correlating with disease duration as would be expected ${ }^{53}$. Studies have also shown a mixture of phases suggesting ongoing injury ${ }^{40}$. While multinuclear pneumocytes and pneumocyte atypia have been reported, definitive viral cytopathic changes do not appear to be a component of COVID19 pathology $40,43,48,52,54,55$. This is not surprising, given that they were not reported in SARS-1 cases, with the exception of a rare case that was in actuality a CMV inclusion instead of SARSrelated $^{28}$. Interestingly, detection of SARS-CoV2-2 virus by in situ hybridization typically shows the presence of virus in early disease, which decreased or was not present in organizing or fibrotic disease ${ }^{56}$. In regard to inflammation, the presence of lymphocytic inflammation was variable and some studies reported a circumferential pattern of distribution around blood vessels ${ }^{40,43}$. Extensive study of the immune components of COVID-related lung disease has been undertaken and is beyond the scope of this pathology-focused paper; however, a variety of changes in both the innate and adaptive immune systems have been reported, some of which appear to be related to viral load ${ }^{57-59}$.

Given that COVID-19 patients frequently had marked abnormalities of the coagulation system and systemic disease progression appeared to be potentially related to endothelial damage, much interest was focused on the vascular changes present in the lungs. Fibrin and platelet thrombi were reported in many cases, including half of the cases in the series of Bryce et al. ${ }^{40,42,43,45,46,48,52,54,60-63}$. Other vascular findings included areas of capillary injury with findings consistent with endothelitis (Fig. 4A, B). This finding was reported in the series of Borczuk et al. and Bryce et al. ${ }^{41,42}$. These lesions were occasionally seen in the presence of only focal hyaline membrane formation, and in some cases, other features of DAD were lacking entirely. Such lesions were not seen in more advanced disease and were felt to likely represent an early finding. The lesions in these series were similar to those reported by Magro et al., who also demonstrated deposition of complement in these foci ${ }^{64}$. Such complement deposition would not necessarily be surprising in a focus of active lung injury and repair; however, the authors also found similar areas of damage in skin lesions, thus lending support to the systemic nature of the disease. Indeed, complement activation as well as endothelial damage have both been previously thought to be related to multi-system organ failure associated with ARDS, a finding that has also been found in COVID-19 as well ${ }^{65-69}$. Other vascular lesions include the presence of vascular congestion and hemangiomatosis-like change reported by DeMichele et al. ${ }^{45}$, and similar lesions were reported by Doglioni et al. in one of the few reports of early-stage disease using cryobiopsy sampling in antemortem cases ${ }^{70}$. "Glomeruloid" proliferations were also reported by Perez-Mies ${ }^{71}$. A series by Ackermann et al. compared seven cases of COVID ARDS with seven H1N1 ARDS and reported that capillary microthrombi were nine times as prevalent in the COVID cases and reported that new vascular growth was 2.7 times as frequent in COVID cases, with particular note of a process described as "intussuceptive angiogenesis" study, as described in the above section on DAD, vascular changes, including fibrin and platelet thrombi, are normally a component of DAD, and vascular proliferation would be an expected response to injury as part of the repair process. Capillary proliferation is not as well documented in the histology literature of $\mathrm{DAD}$, but is reported ${ }^{15}$. Additionally, regulators of angiogenesis have long been known to be present in cases of ARDS and attempted treatment of ARDS with anticoagulants is also well known ${ }^{1,4,69,73,74}$. This point was highlighted in the accompanying editorial to the Ackermann paper authored by Hariri et al., who also point out that the series is a small number of cases that were of differing stages of disease and not corrected for length of illness $^{75}$. As such, while the reported findings of Ackermann et al. are of interest, they are probably more informative to DAD/ARDS as a whole as opposed to being unique to COVID.

Also of interest is the presence of large-vessel thrombi. Pulmonary emboli were reported in six cases in the series of Bryce et al. and were also reported in other series ${ }^{42,52}$. Interestingly, five of the six pulmonary emboli found in the Bryce series were in cases occurring prior to the institution of an anticoagulation protocol. Thrombi were also reported in other organs, particularly the brain ${ }^{42}$. It should be noted that coagulation abnormalities were also a component of SARS-1 and Middle Eastern respiratory syndrome coronavirus (MERS) ${ }^{76}$. While pulmonary microthrombi in acute DAD in particular are likely a component the acute lung injury itself, large emboli and thrombi in other organs are more likely related to altered coagulation status and endothelial injury ${ }^{77,78}$.

While DAD was the most frequently reported finding in COVID19 autopsy cases, AFOP was also reported, albeit more rarely. While some cases of AFOP were reported in small bedside postmortem biopsies, thus raising the issue of sampling in cases of DAD, as discussed in the section on AFOP above, other cases would appear to represent a bona fide AFOP pattern, which would not be surprising in a disease associated with acute lung injury ${ }^{40,79}$.

In summary, the histologic findings in COVID-19 autopsies have consisted primarily of DAD, with a smaller number of cases of AFOP. Whether or not vascular changes may be more frequent or prominent in COVID-related DAD compared with other etiologies is somewhat unclear; however, regardless of whether there is a 
"unique" COVID histology, it is an unprecedented opportunity to study many aspects of DAD/ALI using more sophisticated methodologies than have been previously available, which will hopefully improve our understanding of disease mechanisms and lead to better treatment strategies.

Knowledge gaps exist in regard to earlier phases of disease in patients who do not become severely ill. Limited information from antemortem studies has shown organizing fibrin and features of acute lung injury in one transbronchial biopsy case $e^{80}$ and similar findings were reported in two lung cancer resections discovered to have COVID-19 at the time of operation after the fact and were felt to represent early changes ${ }^{81}$. The series of twelve cryobiopsies by Doglioni et al. taken within 20 days of symptom onset showed only sparse fibrin in a small number of cases, but otherwise showed patchy areas of type-2 pneumocyte hyperplasia and "dilated and hyperplastic interstitial capillaries and venules" but no hyaline membrane formation or features that otherwise fit with DAD, OP, or AFOP ${ }^{70}$.

Another area of future study is the lung pathology of COVID survivors who have persistent radiographic lung abnormalities and decreased pulmonary function. In some of these patients, the radiographic abnormalities have resolved over time, whereas they have thus far persisted in others ${ }^{82}$. This is reflective of the early literature on DAD in regard to resolution and persistence following survival $^{13,14}$. Clinical, radiologic, and pathologic information from patients with persistent lung disease will likely be a key area of study moving forward, as it will be important to understand this phase of the disease in regard to planning treatment and determining whether patients would benefit from treatment with antifibrotic or anti-inflammatory therapy ${ }^{83}$.

\section{CONCLUSIONS}

DAD is the most frequent histology encountered in patients with clinical ARDS, with some cases showing AFOP, AEP, or DAHC. In the majority of cases, etiology is not apparent from the histology alone and clinical correlation is required to help determine the etiology. The outbreak of cases of EVALI and the COVID-19 pandemic in particular has provided an opportunity to increase our knowledge about DAD in particular in regard to mechanisms beyond the histologic findings alone, particularly in regard to the immune components and cellular injury. Cases of AFOP remain relatively rare in comparison with $D A D$ in patients with clinical ARDS. The presence of fibrin in cases otherwise consistent with OP appears to portend an increased incidence of relapse, but a definitive dividing line regarding how much fibrin is needed to separate the two entities has not been firmly established. Conversely, while AFOP was originally thought to perhaps represent an underreported variant of DAD due to similar overall mortality rates, whether cases of DAD with prominent organizing intra-alveolar fibrin and few hyaline membranes compared with more typical cases with extensive hyaline membrane formation are of any significance has not been studied. Cases of EVALI are associated with acute lung-injury patterns and, while vacuolated macrophages have been reported in several cases, the pathology of EVALI is not that of exogenous lipoid pneumonia. Vitamin-E acetate was identified as a culprit in the large 2019 outbreak; however, vaping devices and black-market products in particular contain a large variety of largely unregulated components and cases of EVALI still occur. Finally, most cases of COVID-19 have shown DAD, potentially with a more pronounced vascular component compared with other etiologies. Although a unique "COVID DAD" histology per se does not seem to exist, the COVID19 cases provide an extraordinary opportunity to expand our knowledge about various components of DAD/ALI via novel methods. Continued knowledge gaps into early and long-term disease exist, although the pathology and mechanisms of longterm disease, in particular, are the subject of considerable interest.

\section{REFERENCES}

1. Ware, L. B. Pathophysiology of acute lung injury and the acute respiratory distress syndrome. Semin. Respir. Crit. Care Med. 27, 337-349 (2006).

2. Bernard, G. R. et al. The American-European consensus conference on ARDS. Definitions, mechanisms, relevant outcomes, and clinical trial coordination. Am. J. Respir. Crit Care Med. 149, 818-824 (1994).

3. Ferguson, N. D. et al. The Berlin definition of ARDS: an expanded rationale, justification, and supplementary material. Intensive Care Med. 38, 1573-1582 (2012).

4. Calfee, C. S. et al. Distinct molecular phenotypes of direct vs indirect ARDS in single-center and multicenter studies. Chest 147, 1539-1548 (2015).

5. Carla, A. et al. Acute respiratory distress syndrome subphenotypes and therapy responsive traits among preclinical models: protocol for a systematic review and meta-analysis. Respir. Res. 21, 81 (2020).

6. Spadaro, S. et al. Biomarkers for acute respiratory distress syndrome and prospects for personalised medicine. J. Inflamm. 16, 1 (2019).

7. Riviello, E. D. et al. Hospital incidence and outcomes of the acute respiratory distress syndrome using the Kigali modification of the Berlin definition. Am. J. Respir. Crit. Care Med. 193, 52-59 (2016).

8. Beasley, M. B. The pathologist's approach to acute lung injury. Arch. Pathol. Lab. Med. 134, 719-727 (2010).

9. Hughes, K. T. \& Beasley, M. B. Pulmonary manifestations of acute lung injury: more than just diffuse alveolar damage. Arch. Pathol. Lab. Med. 141, 916-922 (2017).

10. Manganelli, P., Fietta, P., Carotti, M., Pesci, A. \& Salaffi, F. Respiratory system involvement in systemic vasculitides. Clin. Exp. Rheumatol. 24, S48-S59 (2006).

11. Pesci, A. \& Manganelli, P. Respiratory system involvement in antineutrophil cytoplasmic-associated systemic vasculitides: clinical, pathological, radiological and therapeutic considerations. Drugs R D 8, 25-42 (2007).

12. De Giacomi, F., Vassallo, R., Yi, E. S. \& Ryu, J. H. Acute eosinophilic pneumonia. causes, diagnosis, and management. Am. J. Respir. Crit. Care Med. 197, 728-736 (2018).

13. Tomashefski, J. F. Jr Pulmonary pathology of the adult respiratory distress syndrome. Clin. Chest Med. 11, 593-619 (1990).

14. Tomashefski, J. F. Jr Pulmonary pathology of acute respiratory distress syndrome. Clin. Chest Med. 21, 435-466 (2000).

15. Tomashefski, J. F. Jr et al. The pulmonary vascular lesions of the adult respiratory distress syndrome. Am. J. Pathol. 112, 112-126 (1983).

16. Travis, W. D. et al. An official American Thoracic Society/European Respiratory Society statement: update of the international multidisciplinary classification of the idiopathic interstitial pneumonias. Am. J. Respir. Crit. Care Med. 188, 733-748 (2013).

17. Beasley, M. B., Franks, T. J., Galvin, J. R., Gochuico, B. \& Travis, W. D. Acute fibrinous and organizing pneumonia: a histological pattern of lung injury and possible variant of diffuse alveolar damage. Arch. Pathol. Lab. Med. 126, 1064-1070 (2002).

18. Gomes, R. et al. Acute fibrinous and organizing pneumonia: a report of 13 cases in a tertiary university hospital. Medicine 95, e4073 (2016).

19. Damas, C., Morais, A., Moura, C. S. \& Marques, A. Acute fibrinous and organizing pneumonia. Rev. Port. Pneumol. 12, 615-620 (2006).

20. Garcia, B. A., Goede, T. \& Mohammed, T. L. Acute fibrinous organizing pneumonia: a case report and literature review. Curr. Probl. Diagn. Radiol. 44, 469-471 (2015).

21. Hariri, L. P. et al. Acute fibrinous and organizing pneumonia in systemic lupus erythematosus: a case report and review of the literature. Pathol. Int. 60, 755-759 (2010).

22. Heo, J. Y. et al. Acute fibrinous and organizing pneumonia in a patient with HIV infection and Pneumocystis jiroveci pneumonia. Respirology 15, 1259-1261 (2010).

23. Johkoh, T., Fukuoka, J. \& Tanaka, T. Rare idiopathic intestinal pneumonias (IIPs) and histologic patterns in new ATS/ERS multidisciplinary classification of the IIPs. Eur. J. Radiol. 84, 542-546 (2015).

24. Nguyen, L. P. et al. Acute fibrinous and organizing pneumonia associated with allogenic hematopoietic stem cell transplant successfully treated with corticosteroids: a two-patient case series. J Investig. Med. High Impact Case Rep. 4, 2324709616643990 (2016)

25. Otto, C. et al. Acute fibrinous and organizing pneumonia associated with influenza A/H1N1 pneumonia after lung transplantation. BMC Pulm. Med. 13, 30 (2013).

26. Saxena, P. et al. Acute fibrinous and organizing pneumonia: a rare form of nonbacterial pneumonia. Indian J. Crit. Care Med. 20, 245-247 (2016).

27. Valim, V., Rocha, R. H., Couto, R. B., Paixao, T. S. \& Serrano, E. V. Acute fibrinous and organizing pneumonia and undifferentiated connective tissue disease: a case report. Case Rep. Rheumatol. 2012, 549298 (2012).

28. Hwang, D. M. et al. Pulmonary pathology of severe acute respiratory syndrome in Toronto. Mod. Pathol. 18, 1-10 (2005).

29. Yoshinouchi, T., Ohtsuki, Y., Kubo, K. \& Shikata, Y. Clinicopathological study on two types of cryptogenic organizing pneumonitis. Respir. Med. 89, 271-278 (1995). 
30. Nishino, M., Mathai, S. K., Schoenfeld, D., Digumarthy, S. R. \& Kradin, R. L. Clinicopathologic features associated with relapse in cryptogenic organizing pneumonia. Hum. Pathol. 45, 342-351 (2014).

31. Layden, J. E. et al. Pulmonary illness related to E-cigarette use in Illinois and Wisconsin - Final Report. N. Engl. J. Med. 382, 903-916 (2020).

32. Cecchini, M. J. et al. E-cigarette or vaping product use-associated lung injury: a review for pathologists. Arch. Pathol. Lab. Med. 144, 1490-1500 (2020).

33. Matsumoto, $\mathrm{S}$. et al. Dose-dependent pulmonary toxicity of aerosolized vitamin $\mathrm{E}$ acetate. Am. J. Respir. Cell Mol. Biol. 63, 748-757 (2020).

34. Fels Elliott, D. R. et al. Giant cell interstitial pneumonia secondary to cobalt exposure from e-cigarette use. Eur. Respir. J. 54, 1901922 (2019).

35. Niu, S. et al. Significance of Oil-Red-O positive macrophages in bronchoalveolar lavage in diagnosing e-cigarettes or vaping product use-associated lung injury: a case series. Diagn. Cytopathol. 49, 876-884 (2021).

36. Butt, Y. M. et al. Pathology of vaping-associated lung injury. N. Engl. J. Med. 381, 1780-1781 (2019).

37. Mukhopadhyay, S. et al. Lung biopsy findings in severe pulmonary illness associated with e-cigarette use (Vaping). Am. J. Clin. Pathol. 153, 30-39 (2020).

38. Lee, M. H., Cool, C. D. \& Maloney, J. P. Histopathological correlation of acute on chronic eosinophilic pneumonitis caused by vaporized cannabis oil inhalation Chest 159, e137-e139 (2021).

39. Edmonds, P. J., Copeland, C., Conger, A. \& Richmond, B. W. Vaping-induced diffuse alveolar hemorrhage. Respir. Med. Case Rep. 29, 100996 (2020).

40. Borczuk, A. C. Pulmonary pathology of COVID-19: a review of autopsy studies. Curr. Opin. Pulm. Med. 27, 184-192 (2021).

41. Borczuk, A. C. et al. COVID-19 pulmonary pathology: a multi-institutional autopsy cohort from Italy and New York city. Mod. Pathol. 33, 2156-2168 (2020).

42. Bryce, C. et al. Pathophysiology of SARS-CoV-2: the Mount Sinai COVID-19 autopsy experience. Mod. Pathol. 1-12 https://doi.org/10.1038/s41379-021-00793-y (2021).

43. Calabrese, F. et al. Pulmonary pathology and COVID-19: lessons from autopsy. The experience of European pulmonary pathologists. Virchows Arch. 477 359-372 (2020).

44. Chmielik, E. et al. COVID-19 autopsies: a case series from Poland. Pathobiology $\mathbf{8 8}$ 78-87 (2021)

45. De Michele, S. et al. Forty postmortem examinations in COVID-19 patients. Am. J. Clin. Pathol. 154, 748-760 (2020).

46. Elsoukkary, S. S. et al. Autopsy findings in 32 patients with COVID-19: a singleinstitution experience. Pathobiology 88, 56-68 (2021).

47. Falasca, L. et al. Postmortem findings in Italian patients with COVID-19: a descriptive full autopsy study of cases with and without comorbidities. J. Infect. Dis. 222, 1807-1815 (2020).

48. Maiese, A. et al. Autopsy findings in COVID-19-related deaths: a literature review. Forensic Sci. Med. Pathol. 17, 279-296 (2021).

49. Menter, T. et al. Postmortem examination of COVID-19 patients reveals diffuse alveolar damage with severe capillary congestion and variegated findings in lungs and other organs suggesting vascular dysfunction. Histopathology 77, 198-209 (2020)

50. Merdji, H. et al. Histopathological features in fatal COVID-19 acute respiratory distress syndrome. Med. Intensiv. 45, 261-270 (2021).

51. Roden, A. C. et al. The spectrum of histopathologic findings in lungs of patients with fatal coronavirus disease 2019 (COVID-19) infection. Arch. Pathol. Lab. Med. 145, 11-21 (2021).

52. Satturwar, S. et al. Postmortem findings associated with SARS-CoV-2: systematic review and meta-analysis. Am. J. Surg. Pathol. 45, 587-603 (2021).

53. Li, Y. et al. Progression to fibrosing diffuse alveolar damage in a series of 30 minimally invasive autopsies with COVID-19 pneumonia in Wuhan, China. Histopathology 78, 542-555 (2021).

54. Parra-Medina, R., Herrera, S. \& Mejia, J. Systematic review of microthrombi in COVID-19 autopsies. Acta Haematol. https://doi.org/10.1159/000515104 (2021).

55. Hanley, B. et al. Histopathological findings and viral tropism in UK patients with severe fatal COVID-19: a post-mortem study. Lancet Microbe 1, e245-e253 (2020).

56. Schaefer, I. M. et al. In situ detection of SARS-CoV-2 in lungs and airways of patients with COVID-19. Mod. Pathol. 33, 2104-2114 (2020)

57. Grant, R. A. et al. Circuits between infected macrophages and T cells in SARS-CoV2 pneumonia. Nature 590, 635-641 (2021)

58. Melenotte, C. et al. Immune responses during COVID-19 infection. Oncoimmunology 9, 1807836 (2020).

59. Merad, M. \& Martin, J. C. Pathological inflammation in patients with COVID-19: a key role for monocytes and macrophages. Nat. Rev. Immunol. 20, 355-362 (2020).

60. Kommoss, F. K. F. et al. The pathology of severe COVID-19-related lung damage. Dtsch Arztebl Int 117, 500-506 (2020).

61. Rapkiewicz, A. V. et al. Megakaryocytes and platelet-fibrin thrombi characterize multi-organ thrombosis at autopsy in COVID-19: a case series. EClinicalMedicine 24, 100434 (2020).
62. Sadegh Beigee, F. et al. Diffuse alveolar damage and thrombotic microangiopathy are the main histopathological findings in lung tissue biopsy samples of COVID-19 patients. Pathol. Res. Pract. 216, 153228 (2020).

63. Shanmugam, C. et al. COVID-2019 - A comprehensive pathology insight. Pathol. Res. Pract. 216, 153222 (2020).

64. Magro, C. et al. Complement associated microvascular injury and thrombosis in the pathogenesis of severe COVID-19 infection: a report of five cases. Transl. Res. 220, 1-13 (2020).

65. de Nooijer, A. H. et al. Complement activation in the disease course of coronavirus disease 2019 and its effects on clinical outcomes. J. Infect. Dis. 223 214-224 (2021).

66. Li, Q. \& Chen, Z. An update: the emerging evidence of complement involvement in COVID-19. Med. Microbiol. Immunol. 210, 101-109 (2021)

67. Stenmark, K. R. et al. Mechanisms of SARS-CoV-2-induced lung vascular disease: potential role of complement. Pulm. Circ. 11, 20458940211015799 (2021).

68. Bonaventura, A. et al. Endothelial dysfunction and immunothrombosis as key pathogenic mechanisms in COVID-19. Nat. Rev. Immunol. 21, 319-329 (2021).

69. Vassiliou, A. G., Kotanidou, A., Dimopoulou, I. \& Orfanos, S. E. Endothelial damage in acute respiratory distress syndrome. Int. J. Mol. Sci. 21, 8793 (2020)

70. Doglioni, C. et al. Covid-19 interstitial pneumonia: histological and immunohistochemical features on cryobiopsies. Respiration 100, 488-498 (2021).

71. Pérez-Mies, B. et al. Pulmonary vascular proliferation in patients with severe COVID-19: an autopsy study. Thorax https://doi.org/10.1136/thoraxjnl-2020 216714 (2021).

72. Ackermann, M. et al. Pulmonary vascular endothelialitis, thrombosis, and angiogenesis in covid-19. N. Engl. J. Med. 383, 120-128 (2020).

73. Hendrickson, C. M. \& Matthay, M. A. Endothelial biomarkers in human sepsis: pathogenesis and prognosis for ARDS. Pulm. Circ. 8, 2045894018769876 (2018).

74. Huppert, L. A., Matthay, M. A. \& Ware, L. B. Pathogenesis of acute respiratory distress syndrome. Semin. Respir. Crit. Care Med. 40, 31-39 (2019).

75. Hariri, L. \& Hardin, C. C. Covid-19, angiogenesis, and ARDS endotypes. N. Engl. J. Med. 383, 182-183 (2020)

76. Giannis, D., Ziogas, I. A. \& Gianni, P. Coagulation disorders in coronavirus infected patients: COVID-19, SARS-CoV-1, MERS-CoV and lessons from the past. J. Clin. Virol. 127, 104362 (2020)

77. Maruhashi, T. \& Higashi, Y. Pathophysiological association of endothelial dysfunction with fatal outcome in COVID-19. Int. J. Mol. Sci. 22, 5131 (2021).

78. Nicosia, R. F., Ligresti, G., Caporarello, N., Akilesh, S. \& Ribatti, D. COVID-19 vas culopathy: mounting evidence of an indirect mechanism of endothelial injury. Am. J. Pathol. https://doi.org/10.1016/j.ajpath.2021.05.007 (2021).

79. Copin, M. C., Parmentier, E., Duburcq, T., Poissy, J. \& Mathieu, D. Time to consider histologic pattern of lung injury to treat critically ill patients with COVID-19 infection. Intensive Care Med. 46, 1124-1126 (2020).

80. Harkin, T. J. et al. Delayed diagnosis of COVID-19 in a 34-year-old man with atypical presentation. Lancet Respir. Med. 8, 644-646 (2020).

81. Tian, S. et al. Pulmonary pathology of early-phase 2019 novel coronavirus (COVID19) pneumonia in two patients with lung cancer. J. Thorac. Oncol. 15, 700-704 (2020).

82. Wu, X. et al. 3-month, 6-month, 9-month, and 12-month respiratory outcomes in patients following COVID-19-related hospitalisation: a prospective study. Lancet Respir. Med. https://doi.org/10.1016/s2213-2600(21)00174-0 (2021).

83. Myall, K. J. et al. Persistent post-COVID-19 interstitial lung disease. an observational study of corticosteroid treatment. Ann. Am. Thorac. Soc. 18, 799-806 (2021).

\section{AUTHOR CONTRIBUTIONS}

Dr. Beasley is responsible for the design and writing of the paper.

\section{COMPETING INTERESTS}

The author declares no competing interests.

\section{ADDITIONAL INFORMATION}

Correspondence and requests for materials should be addressed to Mary Beth Beasley.

Reprints and permission information is available at http://www.nature.com/ reprints

Publisher's note Springer Nature remains neutral with regard to jurisdictional claims in published maps and institutional affiliations. 